\title{
Exploring the Psychosocial Impact of Wheelchair and Contextual Factors on Quality of Life of People with Neuromuscular Disorders
}

\author{
THAIS POUSADA GARCÍA, PhD ${ }^{1 *}$, BETANIA GROBA GONZÁLEZ, MsC ${ }^{1}$, LAURA NIETO RIVERO, MsC ${ }^{1}$, JAVIER PEREIRA \\ LOUREIRO, $\mathrm{PhD}^{1}$, EMILIANO DÍEZ VILLORIA, $\mathrm{PhD}^{2}$, and ALEJANDRO PAZOS SIERRA, $\mathrm{PhD}^{1}$ \\ ${ }^{1}$ Faculty of Health Science, University of A Coruña, As Xuvias, Coruña, Spain \\ ${ }^{2}$ Instituto Universitario de Integración en la Comunidad (INICO), University of Salamanca, Salamanca, Spain
}

Neuromuscular disorders (NMDs) are a group of heterogeneous diseases that show differences in incidence, hereditary, etiology, prognosis, or functional impairments. Wheelchair use (manual or powered) is influenced by several factors, including personal and contextual factors, and comprehensive evaluation of their impact is required in order to optimize prescription and provision of wheelchairs. The authors therefore assessed the influence of wheelchair use on the quality of life (QoL) of 60 participants with NMD using the Psychosocial Impact of Assistive Devices Scale (PIADS). The Functional Independence Measure (FIM) and a specially developed questionnaire were used to obtain information about contextual factors and participants' activity profile of activities of the participants. The results showed that using a wheelchair has psychosocial benefits, with the main determinants of benefit being type of wheelchair (powered), non-ambulation ability, and independence in mobility. Ensuring a good match between user and assistive technology (AT; e.g., wheelchair), as well as the effectiveness of the particular device, will increase the likelihood that the user will adopt it and use it effectively in daily life. Clinical prescription of AT would be improved by making appropriate use of outcome measures.

Keywords: assistive technology, contextual factors, neuromuscular disorders, outcomes, wheelchair, quality of life

Neuromuscular disorders (NMDs) are a group of diseases that share a high heterogeneity in incidence, hereditary, etiology, prognosis, and functional impairments (Cup et al., 2007; Fowler, Graves, Wetzel, \& Spencer, 2004). Although NMDs vary in severity, the common characteristics are a decrease in muscle strength, limb contractures, spine deformity, and reduced pulmonary function (Fowler et al., 2004). These common problems reduce sufferers' functional capacity and result in progressive loss of physical capacity and participation in physical activities (McDonald, 2002; Vandervelde, Van den Bergh, Goemans, \& Thonnard, 2009). As NMDs progress, they affect the health status, personal autonomy, and independence as well as the needs, expectations, and preferences of the sufferer, who will gradually come to require more assistance from carers and technology to carry out activities of daily living.

The most obvious progressive loss of functionality experienced by people with NMD is related to personal mobility and ambulation (Boström, Nätterlund, \& Ahlström, 2005). Even when people with NMD are still able to walk, the risk of falls and the associated fatigue may compel them to use assistive technology (AT). A wheelchair may function both as a means of

*Address correspondence to: Thais Pousada García, Faculty of Health Science, University of A Coruña. As Xuvias, s/n. P.C. 15006 A Coruña, Spain. Email: tpousada@udc.es enhancing the user's mobility and functional ability and reducing his or her dependence on a caregiver (Muscular Dystrophy Campaign, 2006). Using a wheelchair may allow the person with NMD to carry out a variety of activities, such as self-care, homework, education, work, leisure, or community activities independently; in other words, people with NMD most commonly use wheelchairs (manual or powered) as an AT Device. Used as a device, wheelchairs should enhance the users' quality of life (QoL) and promote greater independence (Muscular Dystrophy Campaign, 2006).

Use of wheelchairs as an AT device has doubled in the last decade, and this form of use continues to increase (Harris \& Sprigle, 2008; Salminen, Brandt, Samuelsson, Töytäri, \& Malmivaara, 2009). Wheelchairs are highly visible signs of disability. Wheelchair users may have more limited ability to carry out activities of daily living than users of other mobility devices, because wheelchair use can increase or decrease independence depending on the user's habits and roles, and can thus be a limiting or a liberating technology (Harris, 2007; Harris \& Sprigle, 2008).

The International Classification of Functioning (ICF; World Health Organization, 2001) assumes that contextual factors (environmental and personal factors) have an important impact on capacity to perform various activities and participation in these activities. The ICF definition of environmental factors includes the individual's physical, social, and attitudinal 
environment, as well as the services to which he or she has access and the systems and policies which influence his or her life. Wheelchair use is influenced by a range of environmental factors, especially those which are strongly related to the accessibility of the physical environment, such as curb cuts, parking, accessible bathrooms, and ramps (Harris, 2007). Environmental factors play a large role in use of mobility devices, because regardless of the quality of the device, it is difficult to be active and participate in social activities if the environment is not accessible or supportive (Salminen et al., 2009).

People with progressive disorders such as NMD vary in the meanings they attribute to ATs. The following personal factors are important influences on how ATs are viewed: the importance attached to controlling symptoms, the importance attributed to fitting in, the value placed on independence, the potential user's values and goals, and whether a device is viewed as a sign of disease progression. Successful integration of an AT device, such as a wheelchair, into a person's life requires consideration of several factors, including the meaning given to the device, his or her expectations of the benefits, the anticipated social cost, etc. (Pape, Kim, \& Weiner, 2002).

Continuing use of an AT is therefore influenced by both environmental and personal (contextual) factors. There is an extensive body of evidence relating abandonment and non-use of assistive devices to a multitude of causes, such as the cost of the devices, the physical skill required to use the device effectively, demographic factors, accessibility problems, and social acceptance (Scherer, 2001; Smith, Seitz, Jansen, \& Rust, 2006). Mobility aids, such as wheelchairs, were the most frequently abandoned devices in a sample of 227 adults with chronic disabilities (Phillips \& Zhao, 1993). This suggests that it is important to measure user satisfaction in order to determine whether a mobility device meets the user's needs.

Wheelchair use is clearly prevalent in people with NMD and acceptance of a wheelchair implies acknowledgement of the progression of the disease; Louise-Bender et al. (2002) asserted that people who have a progressive disorder consider the use of AT as a sign that they are "nearing the end." It is therefore important to investigate the factors that influence wheelchair use, in order to improve the supply system and ensure that there is a good match between user and device so as to minimize the probability of abandonment. A better understanding of the effects of wheelchairs on the QoL of people with NMD is important, particularly in view of the influence of contextual factors on QoL. The impact of an AT on QoL has been defined as "the extent to which it allows a person to enjoy important possibilities in his/her life" (Renwick, Brown, \& Raphael, 1994, p. 35). The two main objectives of this study were therefore (1) to determine the influence of wheelchair use on psychosocial aspects of QoL (e.g., competence, adaptability, and self-esteem) in people with NMD and (2) to determine the influence of contextual factors on QoL of wheelchair users with NMD.

\section{Methods}

\section{Study Design}

This was a cross-sectional study. The descriptive section provides an overview of the sample profile and the characteristics of wheelchairs used by participants. Regression analysis was used to assess the existence and magnitude of causal effects of one or more independent variables upon a dependent variable of interest at a given point in time. Inferential reasoning was used to draw conclusions about possible relationships between the independent and dependent variables.

The work presented here lasted 16 months and was carried out in Galicia, Spain, in collaboration with the Galician Association Against Neuromuscular Diseases (ASEM Galicia). ASEM Galicia is a non-profit organization, consisting of people with NMDs, relatives of people with NMD, and other people who provide support and guidance to people with NMD and their families.

All participants provided written informed consent before being enrolled in the study. The ethical code of ASEM Galicia was followed during this study. Data provided by participants was treated confidentially in compliance with the relevant legislation.

\section{Sample}

The sample was selected from the ASEM Galicia database because there was no other up-to-date epidemiological information available for the region. The database contained details of 338 people with NMD or related pathologies. The following inclusion criteria were used to select the final sample: diagnosed with known or probable NMD, age greater than or equal to 7 years, living in a Galician town, owning or using a wheelchair for personal mobility. This resulted in a final sample of 60 participants.

\section{Survey Instruments}

The primary objective of this research was to assess, in people with NMD, the impact on QoL (measured in terms of competence, adaptability, and self-esteem) of using a wheelchair (manual or powered, not scooter devices). Various independent and dependent variables were selected; Figure 1 shows how they were classified into four categories: Person/User, Activity and Participation, Wheelchair, and Environment.

The following instruments were used to collect data:

- User/wheelchair questionnaire: The research group developed a two-section questionnaire to collect data on personal factors, physical capabilities and participation in activities, and environmental factors (user questionnaire) and features of the participant's wheelchair (wheelchair data sheet).

- Functional Independence Measure (FIM): The FIM is one of the most widely accepted and used instruments for the assessment of rehabilitation outcomes. It is designed to measure the user's capabilities rather than their deficits (Smith, 1996). The questionnaire uses 18 items to assess six aspects of functioning: self-care, sphincter control, mobility, locomotion, communication, and social cognition. Items are also grouped into two domains: motor functioning (13 items) and cognitive functioning (5 items). All items are scored using a 7-point Likert scale ranging from 1 (assistance is needed with every aspect of the activity) to 7 (activity is performed without any support) (Uniform Data System for Medical Rehabilitation, 1997). The maximum possible score is 126 , indicating complete functional 


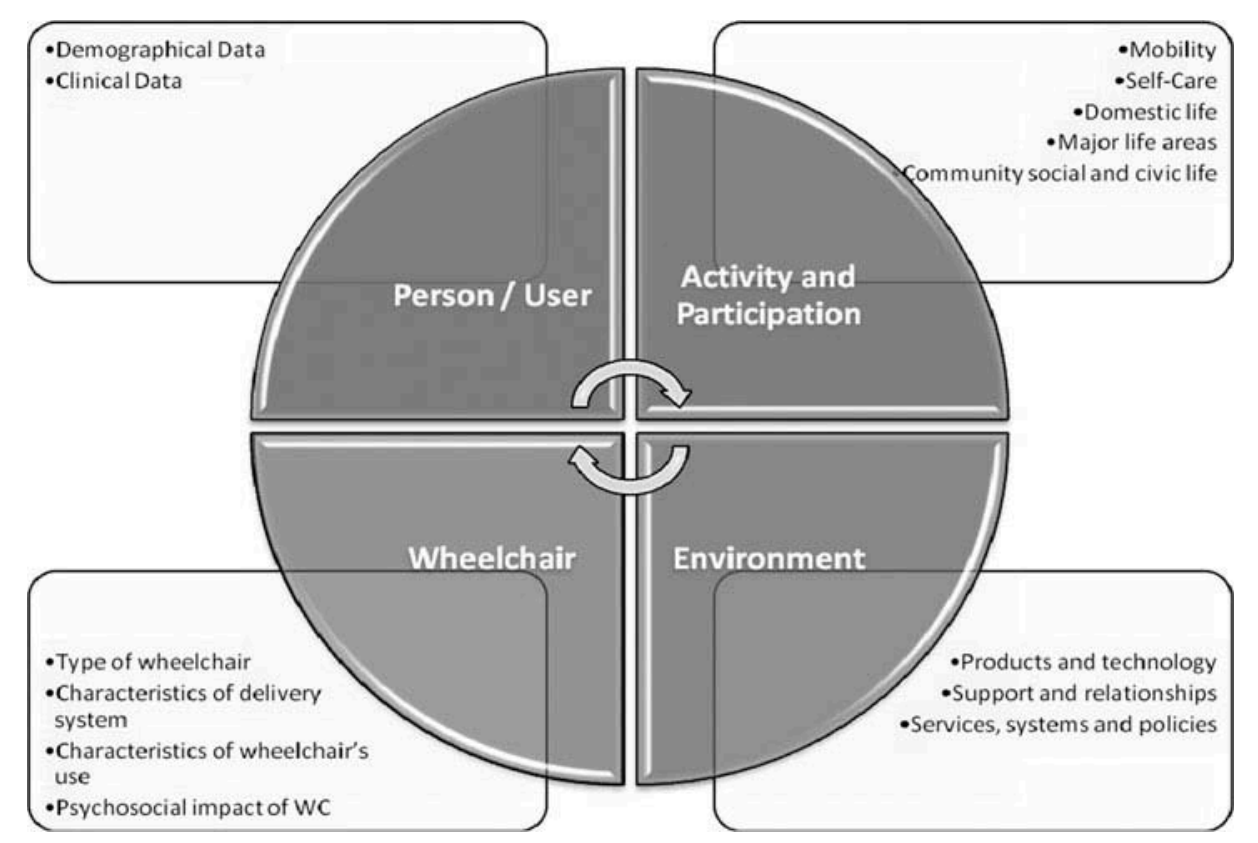

Fig. 1. Classification of variables.

independence, and the lowest score is 18, indicating maximal dependence. The FIM's validity, reliability, and sensitivity to change have been established in several studies (Dodds, Martin, Stolov, \& Deyo, 1993; Linacre, Heinemann, Wright, Granger, \& Hamilton, 1994; Sharrack, 1999). Research on the psychometric properties of a self-report version of the FIM (FIM-SR) in people with NMD showed that it had excellent reliability and validity (Hoffman, Jensen, Abresch, \& Carter, 2005). A validated Spanish version of the FIM was used in this study (Martínez-Martín et al., 2009).

- Psychosocial Impact of Assistive Devices Scale (PIADS): The PIADS was developed to address the need for QoL-related outcome indicators relevant to use of ATs. It has been used to assess the impact of a variety of ATs in various populations (Day \& Jutai, 1996; Devitt, Chau, \& Jutai, 2003; Jutai, Rigby, Ryan, \& Stickel, 2000). It is a self-report questionnaire which assesses the effects of having access to an AT on functional independence, well-being, and QoL. It consists of 26 items grouped into the following three subscales: competence (12), adaptability (6), and self-esteem (8). The scale captures both positive and negative effects using a scale with ranges from -3 (maximal negative impact) to +3 (maximal positive impact); no effect is scored 0 . The validity, reliability, and sensitivity of the PIADS have been described in several publications as being good to excellent (Day \& Jutai, 1996; Jutai \& Gryfe, 1998), and a Spanish version (Quinteiro, 2010) is available.

\section{Procedure}

Participants who met the inclusion criteria were contacted, and data were collected using one of the following three methods:

- Personal interview at the user's home: This was the preferred approach as it allowed the researcher to develop a thorough understanding of contextual factors and improved the reliability of the data. Visits lasted between 1.5 and 2 hours. Data from 38 participants were collected using this method.

- Questionnaires sent by post or e-mail: When a personal interview was not possible or the participant preferred more privacy, the questionnaires were sent to them. Participants who had not returned the forms after 1 month were called to remind them to do so. Data from 21 participants were collected using this method.

- Telephone interview: One user preferred being interviewed over the telephone, given the difficulties in traveling to his home, and establishing an appointment.

To ensure that the correct procedures (adequate interpretation of forms and completion of them in the right order) were followed in all cases and to reduce differences between the three data collection methods, all participants were given a written sheet with precise instructions about the procedure before any data were collected.

\section{Data Analysis}

Descriptive statistics were calculated to provide a profile of participants. Quantitative variables are presented as mean $(M) \pm$ standard deviation $(S D)$, median, and range (minimum and maximum). Qualitative variables are presented as absolute frequency and valid percentage.

Potential relationships between variables were assessed using non-parametric bivariant analysis in view of the sample size. Scores on all the QoL subscales (competence, adaptability, and self-esteem) were treated as separate quantitative variables.

The relationship between variables was measured using Spearman's rho correlation coefficient. Mann-Whitney U-tests were used to compare means for dichotomous qualitative variables, and Kruskal-Wallis tests were used to compare means 
where there were more than two groups or polychotomous characteristics.

The significance level was set at $\alpha=0.05$ for all tests and SPSS version 16 was used to perform all analyses.

\section{Results}

\section{Participant Characteristics}

There were slightly more men $(60 \%)$ than women $(40 \%)$ in the sample and the mean age of participants was 35.15 years $(S D=17.1)$; the most common age category was $36-50$ years old $(n=18)$. Only two types of marital status were reported and there were more single participants $(80 \%)$ than married participants $(20 \%)$.

The sociodemographic characteristics of the sample are shown in Table 1.

Diverse clinical diagnoses were represented in the sample, but the largest subgroup was that of people with muscular dystrophy (MD; 58.3\%). Within this group the most common diagnosis was limb girdle MD (12 cases), followed by Spinal Muscular Atrophy and arthrogryposis, with seven cases each. The time from diagnosis varied from 4 to 53 years. Most participants had been diagnosed in childhood or adolescence, and 10 had been diagnosed during their first year of life. According to Hoffer's classification (Hoffer, Feiwell, Perry, Perry, \& Bonnett, 1973), most of the sample (81.6\%) lacked functional ambulation (nonfunctional ambulation or non-ambulant). Only 11 participants could still walk (functional ambulation - at home or in the community). Only three participants had additional diagnosis and almost the half the total sample was involved in some kind of rehabilitation program (physical therapy, occupational therapy, or speech therapy among others).

The sample was very heterogeneous with respect to physical capabilities and participation in activities. The mean FIM score was $87.6(S D=17.4)$; the mean score on the motor FIM was $53.8(S D=16.5)$, and the mean score on the cognitive FIM was $33.6(S D=3.3)$. The activities with which participants were most likely to require assistance were taking a bath or shower, getting dressed and moving to and from a bed or a chair.

Most of those who were independent with respect to locomotion (assessed using the "location" item of FIM) used electric wheelchairs $(n=26)$ and were classified as having nonfunctional ambulation $(n=31$; . Hoffer et al., 1973). Figure 2 relates locomotor independence to type of wheelchair used. Figure 3 relates locomotor independence to Hoffer ambulation category.

Participants were more independent in carrying out general home management tasks; only eight out of 48 needed someone else's help, but for other activities which made greater physical demands, the number of dependent people (needing some help from the other) increased to $58.3 \%$.

The 49 participants who used a car could transport their wheelchairs in it. Only 23 participants used a car which had been adjusted to facilitate driving, access, or both. Twenty-one participants $(12.6 \%)$ never used public transport, stating that this was because it was not wheelchair friendly.

At the time of the interview, 18 of the 60 users were enrolled in some sort of educational course, and five were working.

The main reasons given for not being in employment were having been declared unfit to work or having a severe disability according to the terms of Spanish disability laws.

Table 1. Sociodemographic characteristics of the participants.

\begin{tabular}{llcc}
\hline Variable & & $N$ & $\%$ \\
\hline Gender & Men & 36 & $60 \%$ \\
Marital status & Women & 24 & $40 \%$ \\
& Single & 48 & $80 \%$ \\
& With partner & 0 & 0 \\
& Married & 12 & $20 \%$ \\
City/town & Divorced & 0 & 0 \\
& Widower & 0 & 0 \\
& City with more than 200,000 inhabitants & 18 & $30 \%$ \\
Education level & 85,000-199,999 inhabitants & 10 & $16.7 \%$ \\
& $40,000-84,999$ inhabitants & 6 & $10 \%$ \\
& City with fewer than 39,999 inhabitants & 26 & $43.3 \%$ \\
& No formal education & 0 & 0 \\
& Primary school & 21 & $35 \%$ \\
Driving license & High school & 15 & $25 \%$ \\
& Bachelor/degree & 11 & $18.3 \%$ \\
Range of age & Job training & 13 & $21.7 \%$ \\
& Yes (\% over 48 persons, $>18$ years) & 14 & $29.2 \%$ \\
& No (\% over 48 persons $>18$ years) & 34 & $70.8 \%$ \\
& Not applicable (age $<18$ years & 12 & $20 \%$ \\
& $7-17$ years & 12 & $20 \%$ \\
& $18-35$ years & 16 & $26.7 \%$ \\
& 36-50 years & 18 & $30 \%$ \\
& $>50$ years & 14 & $23.3 \%$ \\
\hline
\end{tabular}




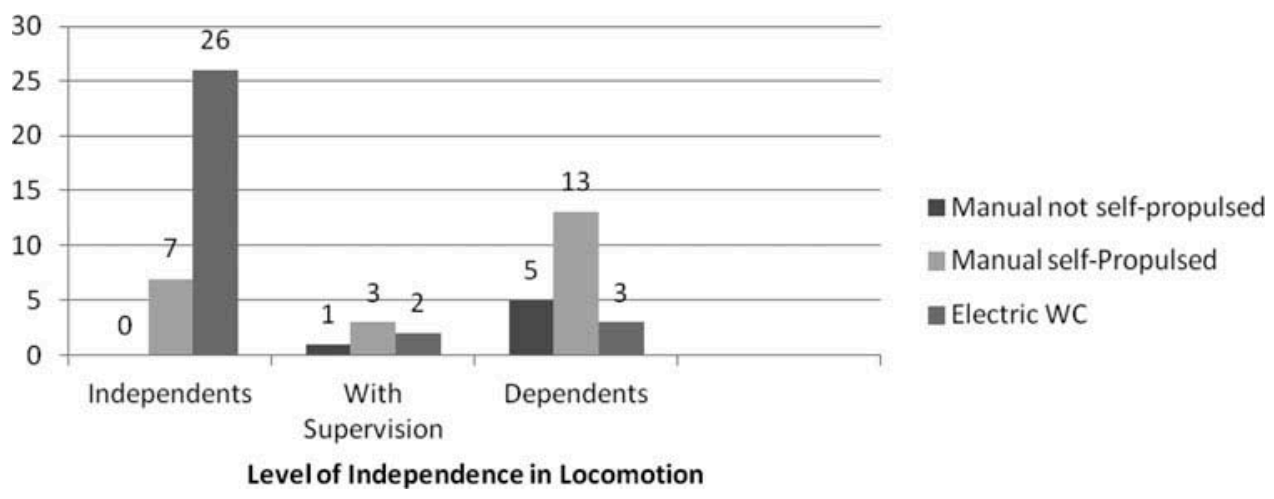

Fig. 2. Level of independence in locomotion (FIM) according to the type of wheelchair.

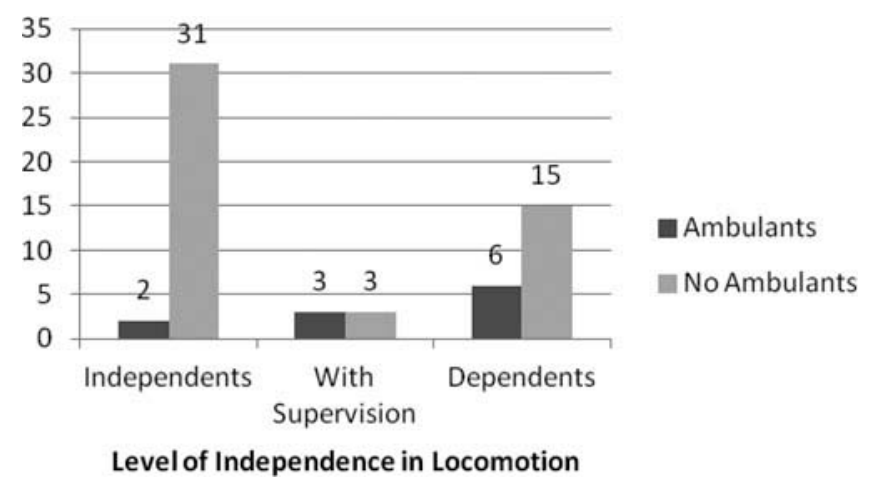

Fig. 3. Level of independence in locomotion (FIM) according to the type of ambulation (Hoffer's classification).

The participants who were regular wheelchair users $(81.6 \%)$ used their wheelchair for leisure activities and in their free time. Little more than half of the sample $(51.7 \%)$ reported participating in four or more different leisure activities; 29 participants were involved in three or fewer leisure activities. The most popular leisure activities were using a computer $(n=27)$ and reading $(n=22)$.

The authors asked about barriers to activities and access in the physical environment and the availability of adjustments or specific measures to improve accessibility. At the interview, 12 participants $(20 \%)$ reported that their home was without architectural barriers to mobility, but 10 had previously adapted their environment to their needs. The room that most frequently presented some type of mobility barrier was the bathroom $(56.6 \%$ of participants reported that their bathroom presented obstacles (especially to use the bath/shower and toilet). The frequency in which AT are used was low or very low because most participants $(60 \%)$ only use one or no products.

Finally, most participants $(81.7 \%)$ were living with relatives or with their partner at the time of the interview, seven were living with non-relatives, and three were living alone. The most common living situation was living with parents (38 participants). Only three participants did not need help from a caregiver; for the vast majority $(75.4 \%)$, the primary caregiver was a relative, but 14 had hired a personal assistant.

\section{Wheelchair Characteristics}

Almost half the sample (48.3\%) used a manual wheelchair. Six participants $(10 \%)$ used a manual wheelchair that was not selfpropelled, whereas 23 participants $(38.3 \%)$ used a self-propelled manual wheelchair. Thirty-one participants (51.7\%) used a powered wheelchair (propelled by a caregiver in one case). It is worth drawing attention to the wide range in the length of time for which the participant had been using a wheelchair (1-37 years).

Only $44.8 \%$ of those who use a manually propelled wheelchair (13 participants) used their wheelchair both indoors and outdoors, but participants with powered wheelchairs were more likely to use them indoors and outdoors $(n=23)$, although some only used their wheelchair outdoors $(n=8)$.

Most participants $(88.3 \%)$ used their main wheelchair on a daily basis ("most of the time" or for "a few hours"), whereas $10 \%$ (five manual wheelchair users, one powered wheelchair user) used it on a weekly basis; only one participant (a manual wheelchair user) reported using the wheelchair as little as several times per month. This low frequency of use (11.7\%) might be due to manual wheelchair users being community or home ambulant. In addition to the normal frequency of use, powered wheelchair users tended only to use their wheelchair outdoors; at home they used an office chair or another device. Problems associated with wheelchair use were assessed using a multiple response question. 
Only $26.7 \%$ of the sample reported that using a wheelchair had not affected their health. The most commonly reported problem was weight gain, affecting 32 people, followed by scoliosis or contractures, $(n=18)$ and pain $(n=14)$. Pressure ulcers $(n=2)$ were a less common problem.

\section{Psychosocial Impact of Wheelchair Use}

The primary objective of this research was to assess the impact of wheelchair use on the QoL of people with NMD. The presentation of results follows the structure of the PIADS, which is organized into three subscales assessing competence, adaptability, and self-esteem.

Wheelchair use had a moderate positive effect on the QoL of people with NMD. The mean PIADS score for competence was $1.06(S D=0.83$; median $=1$; range $=3.67[-0.92-2.75])$. Wheelchair use had a similar impact on adaptability $(M=1.23$, $S D=1.04$; median $=1$; range $=3.83[-0.83-3]$ ). Wheelchair use had least impact on self-esteem $(M=0.92, S D=0.87$; median $=0.82$; range $=3.63[-1-2.63])$.

There was a relationship between impact of wheelchair use and the type of chair used (Table 2); in terms of all three QoL variables, powered wheelchair users reported that wheelchair use had a greater positive impact than did manual wheelchair users. Analysis of locomotor independence (indexed by score on the FIM "locomotion" item) indicated that participants whose wheelchair use increased their independence with respect to personal mobility $(n=33)$ had higher mean scores on all three PIADS dimensions of PIADS than those who needed to be supervised $(n=6)$ or those who receive someone else's help $(n=21)$. Table 3 relates PIADS scores to locomotor independence.

Tables 2-4 show descriptive statistics for all PIADS dimensions (competence, adaptability, and self-esteem) according to wheelchair type, frequency of wheelchair use, and locomotor independence.

\section{Influence of Contextual Factors on the Impact of the Wheelchair}

The influence of contextual factors on the impact of wheelchair use on subjective well-being was assessed using bivariate analysis.

Wheelchair use had a greater positive impact on QoL in participants diagnosed with arthrogryposis (competence $M=1.98$, $p<0.05$; adaptability $M=1.55 p<0.05$ ). Lack of functional ambulation was associated with wheelchair use having a greater positive impact on QoL, especially in terms of adaptability. Participants who needed their wheelchair to get around reported that wheelchair use had a more positive impact on their QoL $(M=1.4)$ than those who were still able to get around without using a wheelchair $(M=0.45)$.

There was a relationship between functional independence in activities of daily living (measured using the FIM) and the impact of wheelchair use on QoL (measured using the three subscales of the PIADS). The strongest correlation between FIM score and impact on QoL related to locomotion. Given that the majority of participants $(n=26)$ who were independent with respect to personal mobility (FIM locomotion $=6$ ) used a powered wheelchair, it is clear that type of wheelchair influences independent locomotion as well as QoL. Mean score on the three PIADS factors was significantly higher in independently mobile participants (competence $M=1.42$; adaptability $M=1.67$; self-esteem $M=1.21$ ) than those who needed help with locomotion (competence $M=0.52$; adaptability $M=0.6$; self-esteem $M=0.5$; $p<0.01)$. The only environmental factor to have a noticeable influence on the impact of wheelchair use on QoL was place of residence. Those living in a private household had significantly lower $(p<0.05)$ self-esteem $(M=0.83)$ than those living in a care facility or residential care facility $(M=1.5)$. Surprisingly, there was no significant association between architectural barriers or accessibility measures and the impact of wheelchair use.

Comparison of wheelchairs and other mobility ATs and QoL indicated that participants who used canes, crutches, or walkers (walking aids) had significantly lower competence $(M=0.13$; $p<0.01)$, adaptability $(M=0.22 ; p<0.05)$ and self-esteem $(M=0.06 ; p<0.01)$ than participants who used another chair as a secondary mobility AT (competence $M=1.19$; adaptability $M=1.38$; self-esteem $M=1.09$ ). In the case of participants who were still able to walk but needed AT for mobility under certain circumstances, the psychosocial impact of wheelchair use was negligible.

Social environment seems to have a greater influence on the psychosocial impact of wheelchair use. Having a professional caregiver rather than relying on a relative was associated with wheelchair use having a greater positive impact on competence $(M=1.44$ versus $M=0.95 ; p<0.05)$, adaptability ( $M=1.73$ versus $M=1.09 ; p<0.05$ ), and self-esteem $(M=1.31$ versus $M=0.81 ; p<0.05)$. Furthermore, the authors found that the more time a relative devoted to taking care of the participant, the greater the benefit to self-esteem relative to the benefits associated with wheelchair use $(r=0.397 ; p<0.01)$. People who need help with personal mobility $(n=41)$ had a

Table 2. Score on PIADS dimensions by type of wheelchair.

\begin{tabular}{|c|c|c|c|c|c|c|}
\hline \multirow[b]{2}{*}{ Type of wheelchair } & \multicolumn{2}{|c|}{ Competence } & \multicolumn{2}{|c|}{ Adaptability } & \multicolumn{2}{|c|}{ Self-esteem } \\
\hline & $M(S D)$ & Median & $M(S D)$ & Median & $M(S D)$ & Median \\
\hline Manual, not self-propelled $(n=6)$ & $0.487(0.71)$ & 0.63 & $0.445(0.41)$ & 0.59 & $0.647(0.76)$ & 0.57 \\
\hline Manual, self-propelled $(n=23)$ & $0.723(0.59)$ & 0.50 & $0.941(0.95)$ & 0.67 & $0.719(0.82)$ & 0.50 \\
\hline Electrically powered $(n=31)$ & $1.412(0.87)$ & 1.67 & $1.590(1.07)$ & 1.67 & $1.115(0.91)$ & 1.00 \\
\hline
\end{tabular}


Table 3. Scores on PIADS dimensions by frequency of wheelchair use.

\begin{tabular}{|c|c|c|c|c|c|c|}
\hline \multirow[b]{2}{*}{ Frequency of use } & \multicolumn{2}{|c|}{ Competence } & \multicolumn{2}{|c|}{ Adaptability } & \multicolumn{2}{|c|}{ Self-esteem } \\
\hline & $M(S D)$ & Median & $M(S D)$ & Median & $M(S D)$ & Median \\
\hline Daily $(n=53)$ & $1.111(0.84)$ & 1 & $1.293(1.04)$ & 1 & $0.986(0.87)$ & 0.88 \\
\hline Less than daily $(n=7)$ & $0.631(0.72)$ & 0.5 & $0.714(0.99)$ & 0.17 & $0.43(0.82)$ & 0.25 \\
\hline
\end{tabular}

Table 4. Scores of dimensions of PIADS according to the independence in locomotion.

\begin{tabular}{|c|c|c|c|c|c|c|}
\hline \multirow[b]{2}{*}{ Locomotor independence* } & \multicolumn{2}{|c|}{ Competence } & \multicolumn{2}{|c|}{ Adaptability } & \multicolumn{2}{|c|}{ Self-esteem } \\
\hline & $M(S D)$ & Median & $M(S D)$ & Median & $M(S D)$ & Median \\
\hline Independent $(n=33)$ & $1.41(0.77)$ & 1.58 & $1.667(1.04)$ & 1.67 & $1.21(0.88)$ & 1.13 \\
\hline With supervision $(n=6)$ & $0.902(0.96)$ & 0.92 & $0.973(1.01)$ & 1 & $0.73(1.05)$ & 0.57 \\
\hline With any human support $(n=21)$ & $0.520(0.58)$ & 0.5 & $0.603(0.72)$ & 0.33 & $0.507(0.64)$ & 0.5 \\
\hline
\end{tabular}

Note: *According to results from item locomotion in FIM scale (independent $=6$ or 7 points).

lower mean competence score $(M=0.67)$ than those who did $\operatorname{not}(M=1.18 ; p<0.05)$

Finally, the authors found that the main influence on the psychosocial impact of wheelchair use was the type of wheelchair used. As can be seen in Table 2, competence and adaptability were significantly lower in participants who used a manual wheelchair (competence $M=0.67$; adaptability $M=0.84$ ) than those who used a powered wheelchair (competence $M=1.41, p<0.001$; adaptability $M=1.59, p<0.01$ ). Although self-esteem was lower in manual wheelchair users, the difference was only marginally significant $(p=0.06)$.

The authors also detected an effect of experience on wheelchair use. Years since acquisition of the first wheelchair and number of wheelchairs used had a significantly higher impact on competence, adaptability, and self-esteem in powered wheelchair users than manual wheelchair users. In other words, users of powered wheelchairs have more experience in using wheelchairs, and more experienced wheelchair users derive a greater benefit from using a wheelchair. The context in which the wheelchair is used had a significant influence on competence; the positive impact on competence was greater for participants who used their wheelchairs both indoors and outdoors $(n=36 ; M=1.23)$ than those who used a wheelchair in only one context $(n=24$, $M=0.58)$. Ability to use the wheelchair in several different contexts may determine whether using the wheelchair has a positive impact on the user's QoL.

\section{Discussion}

This study was a comprehensive investigation of the impact of wheelchair use on the QoL of people with NMD, which took into account socio-demographic and clinical factors, contextual factors such as wheelchair characteristics, and the activity profile of participants. QoL was evaluated using a well-known instrument for the assessment of the impact of ATs, the PIADS, which is intended mainly for use as a measure of how psychological wellbeing is related to use of a particular device. Overall, the results showed that wheelchair use has a positive psychosocial impact on the QoL of people with NMD.

\section{The Role of the Wheelchair and Its Impact on QoL}

The progressive and chronic nature of NMDs results in loss of functional capacity to perform certain activities, including those that require personal mobility. It is clearly necessary to prescribe wheelchairs for individuals whose ability to walk has been reduced or lost due to the disease (Boström et al., 2005; Muscular Dystrophy Campaign, 2006; Natterlund \& Ahlstrom, 2001; Vandervelde et al., 2009). This is an inevitable and delicate situation for several reasons:

- Prescription of a wheelchair is an indication that the disease has progressed. A period of adaptation to the device is therefore necessary, and the way in which the adaptation process is handled may influence the meaning the user assigns to the device, which may have implications for subsequent usage.

- During the initial adaptation phase, users frequently report feeling a loss of control over their own capacities, performance, and environment (Federación ASEM, 2008).

- Becoming a wheelchair user results in a change in social image; the stigma associated with wheelchairs persists even today, and using a wheelchair is a clear sign of disability.

- Choosing the appropriate moment to prescribe a wheelchair is a delicate process; if someone receives a wheelchair too early in the course of the disease and gets used to relying on it for personal mobility, there will be a more rapid loss of physical ability to walk. Conversely, if a wheelchair is not supplied until too late, the individual may already have suffered a significant reduction in independence (Muscular Dystrophy Campaign, 2006).

When asked to select their main reason for using a wheelchair, the most popular choice among participants in this study was that it offered them the opportunity to leave the home (51 participants); a reduction in the fatigue associated with movement was the second most popular option (38 participants). Only two participants reported that while using their wheelchair they "felt less shy" or that "the device helps relieve the pain." 
The PIADS scores demonstrated that wheelchair use was associated with a positive impact on three aspects of psychosocial functioning: competence, adaptability, and self-esteem. The greatest positive impact of wheelchair use was on adaptability, as in studies by Demmers et al. (2002) and Devitt et al. (2002). This finding suggests that the empowerment to participate actively associated with wheelchair use is the most important factor for satisfaction of users with the wheelchair. The smallest impact of wheelchair use was on self-esteem, which was also consistent with earlier studies (Demmers et al., 2002; Devit et al., 2002). Devitt et al. (2002) suggested that self-esteem may be negatively affected by the progressive, unpredictable nature of NMDs. Additionally, wheelchairs represent a visible sign of disability and are associated with social stigma that may have a negative influence on users' self-esteem.

Even when a wheelchair is absolutely necessary to mobility, wheelchair use does not necessarily have an impact (positive or negative) on the QoL of a person with NMD. Some factors, such as low usage frequency and limited walking ability, had very little influence on how wheelchair use affected the user's well-being (PIADS scores close to 0 ). These findings suggest that a period of adaptation to the device, during which it is integrated into the user's lifestyle, might increase its positive impact on QoL. Louise-Bender et al. (2002) argued that experiences during the adaptation phase not only determined acceptance of increased disability, but also the meaning attributed to the AT and the extent to which it was integrated into users' lives.

\section{Influence of Contextual Factors on the Psychosocial Impact of the Wheelchair}

The influence of certain variables on the impact of wheelchair use on QoL was also explored in this research. These variables included personal factors, activities, participation in social activities, environmental factors, and wheelchair characteristics. All these factors are potential moderators of the strength and direction of the association between wheelchair use and its psychosocial impact, including impact on the QoL of people with NMD.

Age at diagnosis was negatively correlated with self-esteem, that is, the younger a person was at the time of diagnosis, the more likely he or she was to have accepted the diagnosis, acknowledged the need to use a wheelchair, and believe that the device had a positive effect on his or her emotional wellbeing. This may be due to better psychosocial adaptation to deteriorating physical capacity in diseases with slow progression (Boström et al., 2005; Louise-Bender et al., 2002) and appears to corroborate Louise-Bender et al.'s (2002) hypothesis that the effectiveness of an AT is determined by the user's adaptation to or acceptance of his or her disease. The authors also observed that using a powered wheelchair had a more positive impact on the QoL of young participants than using a manual wheelchair, a result which supports previous recommendations that children and young people should be prescribed powered wheelchairs (Butler, 1986; Butler, Okamoto, \& McKay, 1984; Jones, McEwen, \& Hansen, 2003; Tefft, Guerette, \& Furumasu, 1999).

Regarding the diagnostic group, participants with Steinert myotonic dystrophy syndrome had lower scores on all PIADS subscales than participants with other NMDs; this might be explained by characteristics of the disease, such as apathy, depression, indifference, and negligence, which are also associated with difficulties in adapting to ATs (Asociación Francesa contra las miopatías, 2000).

Level of ambulation has been shown to have a marked influence on adaptability (Hoffer et al., 1973). It follows from this that wheelchairs facilitate participation in social activities, because they give users without functional ambulation independent mobility, which opens up a wider range of activities and occupations.

Locomotor independence, assessed with the FIM "locomotion" item, was reported to be one of the main determinants of positive impact of a device on QoL in a study of people with multiple sclerosis (Devitt et al., 2002). The sense of freedom that wheelchair use gives contributes to users' well-being, selfesteem, and self-confidence, and increases their interest in new activities and ability to take advantage of opportunities, as well as their sense of competence and efficiency (Devitt et al., 2003).

Using a wheelchair to carry out activities of daily living was associated with much greater independence in such activities, but this was not reflected in a difference in mean PIADS scores. This finding is consistent with other studies, including that of Nätterlund and Alhström (2002), who argued that personal autonomy in activities of daily living was not be a good predictor of QoL. Additionally, Póveda et al. (1998) found that perceived satisfaction with AT was not associated with the ability to carry out activities of daily living independently. Therefore, overall personal independence does not appear to influence the magnitude of positive effects on well-being which are ascribed to use of a wheelchair.

Environmental factors play an important role in the use of AT for personal mobility because getting around will be harder if the environment is not accessible or supportive, regardless of the quality and suitability of mobility AT (Harris, 2007; Salminen et al., 2009). Nonetheless, in this study the authors found no significant differences in competence and adaptability scores associated with the presence of environmental adaptations or architectural barriers. The data did not allow us to evaluate effects of physical aspects of users' home environments.

Social environment has a strong influence on how people with a disability interpret their experiences (Scherer, 2001). In general the participants in this research had good and supportive relationships with relatives and their main caregiver. Such support is very important, not only to carrying out activities of daily living, but because it contributes to the person's sense of control, well-being, and QoL (Boyer, Drame, Morrone, \& Novella, 2006; Boyer, Novella et al., 2006; Im, Lee, Moon, Park, \& Park, 2010; Vega, 2007). In this study the authors found that social context had a greater influence on the impact of wheelchair use than the physical environment. It appears that having support from caregivers is more important to deriving benefit from a wheelchair than lack of environmental barriers (Warms, Belza, \& Whitney, 2007).

Analysis of associations between wheelchair characteristics and PIADS scores indicated that impact on self-esteem was not related to device variables. Competence was the PIADS dimension for which there was the highest number of significant interactions, indicating that the characteristics and usage 
profile of the wheelchair influence its impact on the productivity, efficiency, and fulfillment of users with NMD.

Despite the obvious advantages of the powered wheelchair and the various studies assessing its effectiveness (Davies, De Souza, \& Frank, 2003; Evans, Neophytou, De Souza, \& Frank, 2007; Liu, Mineo, Hanayama, Fujiwara, \& Chino, 2003; Richardson \& Frank, 2009), to date there has been no empirical demonstration that it offers more benefits to users than a manual wheelchair. Furthermore there has been no research specifically on wheelchair use in people with NMD. In this study use of a powered wheelchair was one of the main factors positively associated with greater impact of wheelchair use on QoL. This finding is consistent with the results of other studies of people with neurodegenerative diseases, such as multiple sclerosis (Devitt et al., 2003) and amyotrophic lateral sclerosis (Jutai \& Gryfe, 1998). This study confirmed the conclusions of earlier research (Davies et al., 2003; Evans et al., 2007; Frank, Ward, Orwell, McCullagh, \& Belcher, 2000) which indicated that using a powered wheelchair improved independence with respect to mobility and social interaction and also improved the comfort and QoL of people with NMD.

\section{Limitations}

The findings of this study are subject to several limitations. First, the small sample size is an important limitation which limits the generalizability of the results and means that they should be interpreted somewhat cautiously. The absence of an up-to-date, official local database on people with NMD made it difficult to ensure that the sample was representative of the population of people with NMD and also restricts the generalizability of the findings. The small sample size also restricted the analytic techniques that could be used, in particular the opportunities for parametric analyses and structural equation modeling.

Second, some potentially relevant variables such as changes in mood (depression or anxiety), economic factors, social resources, and level of medical support were not controlled or measured and may have acted as confounding factors, so the findings should be interpreted cautiously.

Finally, during the administration of the assessment instruments, the authors of this article encountered a problem with the PIADS. The authors of the PIADS recommend that the complete definition of an item should only be offered a respondent if he or she does not understand the original phrasing; however the authors of this article found that the first few participants had difficulty understanding the PIADS items in their original form and therefore took the decision to administer the PIADS using complete definitions of all the items.

\section{Conclusion}

This study provides important information on the psychosocial impact of wheelchairs and the contextual factors which affect the impact of wheelchair use on the QoL of people with NMD. One of the more significant findings to emerge from this study is that wheelchair use was associated with positive changes in QoL, especially for powered wheelchairs; the authors therefore recommend that powered chairs should be made available to people with NMD in clinical practice and that where necessary people with NMD should receive financial assistance to enable them to access an appropriate mobility device. More broadly, the findings from research of this type can be used to improve rehabilitation programs. Taken together, the results suggest for people with NMD successful adoption of a wheelchair, i.e., cases where starting to use a wheelchair has a beneficial impact on QoL is related to a complex set of factors. Wheelchair prescription should therefore be part of a comprehensive intervention program that includes a comprehensive assessment of factors associated with successful adoption, training in use of the device, assessment of the user's physical and social context, and exhaustive follow-up to ensure that the device remains appropriate to the changing needs of the user. This approach depends on coordinated provision of health and social services to ensure continuity of care and effective intervention. The cost effectiveness of a mobility device depends on the extent to which it increases the user's well-being and the occupational choices available to him or her. The results of this study suggest that prescribing practice should take into account the potentially superior impact of a powered wheelchair, relative to a manual wheelchair, on the QoL of people with NMD.

Finally, the results of this exploratory study indicate that measuring the outcomes of AT adoption using measures such as the PIADS and FIM could improve the efficiency of interventions and increase their beneficial impact on the QoL of people who receive new assistive devices. Outcome evaluation could be used to document the success of AT prescription by public health services. Future research should therefore concentrate on measuring the impact of ATs on different populations of people with disabilities to improve their value and scope.

\section{References}

Asociación Francesa contra las miopatías. (2000). La distrofia miotónica de steinert. ficha técnica [Steinertmyotonic dystrophy. Technical specs]. Barcelona, Spain: Asociación Española contra las enfermedades neuromusculares.

Boström, K., Nätterlund, B. S., \& Ahlström, G. (2005). Sickness impact in people with muscular dystrophy: A longitudinal study over 10 years. Clinical Rehabilitation, 19(6), 686-694. doi:10.1191/ $0269215505 \mathrm{cr} 866 \mathrm{oa}$

Boyer, F., Drame, M., Morrone, I., \& Novella, J.-L. (2006). Factors relating to carer burden for families of persons with muscular dystrophy. Journal of Rehabilitation Medicine, 38, 309-315. doi:10.1080/16501970600731578

Boyer, F., Novella, J.-L., Coulon, J.-M., Delmer, F., Morrone, I., Lemoussu, N., . . Étienne, J.-C. (2006). Les aidants familiaux des personnes myopathes: Liaisons entre charge de soins, qualité de vie et santé mentale. Annales De Réadaptation Et De Médecine Physique, 49(1), 16-22. doi:10.1016/j.annrmp.2005.08.001

Butler, C. (1986). Effects of powered mobility on self-initiated behaviors of very young children with locomotor disability. Developmental Medicine and Child Neurology, 28(3), 325-332. doi:10.1111/ j.1469-8749.1986.tb03881.x

Butler, C., Okamoto, G. A., \& McKay, T. M. (1984). Motorized wheelchair driving by disabled children. Archives of Physical Medicine and Rehabilitation, 65(2), 95-97.

Cup, E. H., Pieterse, A. J., Knuijt, S., Hendricks, H. T., Van Engelen, B. G. M., Oostendorp, R. A. B., \& Van Der Wilt, G.-J. (2007). Referral of patients with neuromuscular disease to occupational therapy, physical therapy and speech therapy: Usual practice versus multidisciplinary 
advice. Disability \& Rehabilitation, 29(9), 717-726. doi:10.1080/ 09638280600926702

Davies, A., De Souza, L. H., \& Frank, A. O. (2003). Changes in the quality of life in severely disabled people following provision of powered indoor/outdoor chairs. Disability \& Rehabilitation, 25(6), 286-290. doi:10.1080/0963828021000043734

Day, H., \& Jutai, J. (1996). Measuring the psychosocial impact of assistive devices: The PIADS. Canadian Journal of Rehabilitation, 9, 159-168.

Devitt, R., Chau, B., \& Jutai, J. W. (2003). The Effect of Wheelchair Use on the Quality of Life of Persons with Multiple Sclerosis. Occupational Therapy in Health Care, 17(3/4), 63-79.

Demers, L., Monette, M., Lapierre, Y., et al. (2002). Reliability, validity, and applicability of the Quebec User Evaluation of Satisfaction with assistive Technology (QUEST 2.0) for adults with multiple sclerosis. Disabil Rehabil, 24, 21-30.

Devitt, R., Chau, B., \& Jutai, J. (2003). The effect of wheelchair use on the quality of life of persons with multiple sclerosis. Occupational Therapy in Health Care, 17(3/4), 63-79.

Dodds, T. A., Martin, D. P., Stolov, W. C., \& Deyo, R. A. (1993). A validation of the functional independence measurement and its performance among rehabilitation inpatients. Archives of Physical Medicine and Rehabilitation, 74(5), 531-536. doi:10.1016/0003-9993(93)90119-U

Evans, S., Neophytou, C., De Souza, L., \& Frank, A. O. (2007). Young people's experiences using electric powered indoor - outdoor wheelchairs (EPIOCs): Potential for enhancing users' development? Disability \& Rehabilitation, 29(16), 1281-1294. doi:10.1080/09638280600 964406

Federación ASEM. (2008). Guía de enfermedades neuromusculares. información y apoyo a las familias [Guide to neuromuscular diseases. Information and support for families] (1st Ed.). Jaen, Spain: Formación Alcalá.

Fowler, E. G., Graves, M. C., Wetzel, G. T., \& Spencer, M. J. (2004). Pilot trial of albuterol in duchenne and becker muscular dystrophy. Neurology, 62(6), 1006-1008. doi:10.1212/01.WNL. $0000118530.71646 .0 \mathrm{~F}$

Frank, A. O., Ward, J., Orwell, N. J., McCullagh, C., \& Belcher, M. (2000). Introduction of a new NHS electric-powered indoor/ outdoor chair (EPIOC) service: Benefits, risks and implications for prescribers. Clinical Rehabilitation, 14(6), 665-673. doi:10.1191/ $0269215500 \mathrm{cr} 376 \mathrm{oa}$

Harris, F. (2007). Conceptual issues in the measurement of participation among wheeled mobility device users. Disability \& Rehabilitation: Assistive Technology, 2(3), 137-148. doi:10.1080/ 17483100701374363

Harris, F., \& Sprigle, S. (2008). Outcomes measurement of a wheelchair intervention. Disability \& Rehabilitation: Assistive Technology, 3(4), 171-180. doi:10.1080/17483100701869784

Hoffer, M. M., Feiwell, E., Perry, R., Perry, J., \& Bonnett, C. (1973). Functional ambulation in patients with myelomeningocele. American Journal of Bone and Joint Surgery, 55(1), 137-148.

Hoffman, A. J., Jensen, M. P., Abresch, R. T., \& Carter, G. T. (2005). Chronic pain in persons with neuromuscular disease. Physical Medicine and Rehabilitation Clinics of North America, 16(4), 1099-1112. doi:10.1016/j.pmr.2005.08.015

Im, S. H., Lee, S. C., Moon, J. H., Park, E. S., \& Park, Y. G. (2010). Quality of life for primary caregivers of muscular dystrophy patients in south korea. Chinese Medical Journal, 123(4), 452-457.

Jones, M. A., McEwen, I. R., \& Hansen, L. (2003). Use of power mobility for a young child with spinal muscular atrophy. Physical Therapy, 83(3), 253-262.

Jutai, J., \& Gryfe, P. (1998). Psychological factors in the assessment of assistive technologies. Proceedings of RESNA '98, 54-65.

Jutai, J., Rigby, P., Ryan, S., \& Stickel, S. (2000). Psychosocial impact of electronic aids to daily living. Assistive Technology, 12(2), 123-131. doi: 10.1080/10400435.2000.10132018

Linacre, J. M., Heinemann, A. W., Wright, B. D., Granger, C. V., \& Hamilton, B. B. (1994). The structure and stability of the functional independence measure. Archives of Physical Medicine and Rehabilitation, 75(2), $127-132$.

Liu, M., Mineo, K., Hanayama, K., Fujiwara, T., \& Chino, N. (2003). Practical problems and management of seating through the clinical stages of Duchenne's muscular dystrophy. Archives of Physical Medicine and Rehabilitation, 84(6), 818-824. doi:10.1016/ S0003-9993(02)04953-5

Martínez-Martín, P., Fernández-Mayoralas, G., Frades-Payo, B., Rojo-Pérez, F., Petidier, R., Rodríguez-Rodríguez, V., . . . De Pedro Cuesta, J. (2009). Validación de la escala de independenciafuncional [Validation of the functional independence scale]. Gaceta Sanitaria, 23(1), 49-54. doi:10.1016/j.gaceta.2008.06.007

McDonald, C. M. (2002). Physical activity, health impairments and disability in neuromuscular disease. American Journal of Physical Medicine \& Rehabilitation, 81, S108-120. doi:10.1097/ 00002060-200211001-00012

Muscular Dystrophy Campaign. (2006). Wheelchair provision for children and adults with muscular dystrophy and other neuromuscular conditions (1st Ed.). London, UK: Muscular Dystrophy Campaign.

Natterlund, B., \& Ahlstrom, G. (2001). Activities of daily living and quality of life in persons with muscular dystrophy. $J$ Rehabil Med, 33(5), 206-211.

Pape, T. L.-B., Kim, J., \& Weiner, B. (2002). The shaping of individual meanings assigned to assistive technology: A review of personal factors. Disability \& Rehabilitation, 24(1-3), 5-20. doi:10.1080/ 09638280110066235

Phillips, B., \& Zhao, H. (1993). Predictors of assistive technology abandonment. Assistive Technology, 5(1), 36-45. doi:10.1080/ 10400435.1993.10132205

Poveda, R., Lafuente, R., Sánchez-Lacuesta, J., Viosca, E., Prat, J., Belda, J. M. et al. (1998). Problemática de los usuarios de sillas de ruedas en España (1st Ed.). Valencia, Spain: Instituto de Biomecánica de Valencia.

Quinteiro, M. V. (2010). Impacto de las ayudas técnicas de acceso al ordenador en el aprendizaje de materias informáticas mediante cursos de enseñanza asistida por ordenador (e.a.o.) de alumnos con discapacidad en los miembros superiores [Impact of assistive technologies for computer access on learning about computersof students with disabilities in the upper limbs using computerassisted instruction (CAI)] (Unpublished doctoral dissertation). Universidad Nacional de Educación a Distancia-UNED, Madrid, Spain.

Renwick, R., Brown, I., \& Raphael, D. (1994). Quality of life: Linking a conceptual approach to service provisión. Journal of Developmental Disabilities, 3, 32-44.

Richardson, M., \& Frank, A. O. (2009). Electric powered wheelchairs for those with muscular dystrophy: Problems of posture, pain and deformity. Disability \& Rehabilitation: Assistive Technology, 4(3), 181-188. doi:10.1080/17483100802543114

Salminen, A. L., Brandt, A., Samuelsson, K., Töytäri, O., \& Malmivaara, A. (2009). Mobility devices to promote activity and participation: A systematic review. Journal of Rehabilitation Medicine, 41(9), 697-706. doi:10.2340/16501977-0427

Scherer, M. J. (2001). Assistive technology matching device and consumer for successful rehabilitation. Washington, DC: American Psychological Association.

Sharrack, B. (1999). The psychometric properties of clinical rating scales used in multiple sclerosis. Brain, 122(1), 141-159. doi:10.1093/brain/ 122.1.141

Smith, R. O. (1996). Measuring the outcomes of assistive technology: Challenge and innovation. Assistive Technology, 8(2), 71-81. doi:10.1080/10400435.1996.10132277

Smith, R. O., Seitz, J., Jansen, C., \& Rust, K. L. (2006). ATOMS project technical report-models and taxonomies relating to assistive technology. Retrieved from http://www.r2d2.uwm.edu/atoms/archive/ technicalreports/fieldscans/tr-fs-taxonomiesmodels.html 
Natterlund, B. (2001). Activities of daily living and quality of life in persons with muscular dystrophy. Journal of Rehabilitation Medicine, 33(5), 206-211. doi:10.1080/165019701750419590

Tefft, D., Guerette, P., \& Furumasu, J. (1999). Cognitive predictors of young children's readiness for powered mobility. Developmental Medicine \& Child Neurology, 41(10), 665-670. doi:10.1017/S00121622990 01371

Uniform Data System for Medical Rehabilitation. (1997). Functional independence measure, version 5.1. New York, UK: Buffalo General Hospital.

Vandervelde, L., Van den Bergh, P. Y. K., Goemans, N., \& Thonnard, J.-L. (2009). Activity limitations in patients with neuromuscular disorders: A responsiveness study of the ACTIVLIM questionnaire. Neuromuscular Disorders, 19(2), 99-103. doi:10.1016/j.nmd.2008. 11.004

Vega, E. (2007). Daño cerebral adquirido. La discapacidad invisible [Acquired brain injury. The hidden disability]. Perfiles, 234, 6-10.

Warms, C. A., Belza, B. L., \& Whitney, J. D. (2007). Correlates of physical activity in adults with mobility limitations. Family \& Community Health, 30(2), SS5-16. doi:10.1097/01.FCH.0000264876.429 45.e4

World Health Organization. (2001). International classification of functioning, disability and health - ICF (1st Ed.). Geneva, Switzerland: Author. 
Copyright of Assistive Technology is the property of Taylor \& Francis Ltd and its content may not be copied or emailed to multiple sites or posted to a listserv without the copyright holder's express written permission. However, users may print, download, or email articles for individual use. 